\title{
Analysis of Visible and Invisible Image Watermarking - A Review
}

\author{
Swati Dhiman \\ EEE dept. \\ Arni University \\ Kathgarh (H.P), India
}

\author{
Onkar Singh \\ ECE dept. \\ Arni University \\ Kathgarh (H.P), India
}

\begin{abstract}
As the digital data is transferred over the internet which may harm the digital data like tampering of data etc. Therefore the need of digital data protection has been increased with the advancement in the technology. Watermarking is a technique used to protect the digital data. Digital watermarking is a technique by using which user can get the copyright of its product which prevents the data from tampering. Many techniques are available for video watermarking like Discrete Wavelet Transform, Least Significant Bit Technique etc. [2].The properties of digital watermarking are for copy control, In fingerprinting, For identification of ownership, For Authentication, Monitoring of digital video broadcast, In video tagging etc. [1]. The techniques of video watermarking that have been introduced till date are studied and comparison between all of them is made out to find the best efficient technique. The analysis of the results is done and the implementation is done using the MATLAB software
\end{abstract}

\section{Keywords}

Watermarking, video watermarking, security, DWT, DCT, Robustness, perceptibility

\section{INTRODUCTION}

Digital watermarking is a technique which is used to hide the digital data behind a data. Digital watermarking is a type of steganography. Digital watermarking follows the steganography and hide the digital data behind other data but in this source image and hidden image both has the highest preference. The concept of digital watermarking comes from the concept of image watermarking where the copyright identification is appended on the host image for the purpose of security of image [2].

Once a watermark is embedded on digital data it can be removed anytime according to the need. The process of image watermarking is different from image watermarking. The process of Digital watermarking has two main principles:

\section{Embedding process \\ 2. Extraction Process}

\section{Embedding Process}

This contains the selection of watermark which is going to embed on the digital data. The selection of watermark depends upon the kind of data it is, whether it is original data or compressed data and whether the watermark is visible or not [3].

\section{Extraction Process}

In this process to demonstrate or show the concern of copyright on the data and to make sure that the purpose of watermarking has been achieved the watermark is extracted from the watermarked digital data. The extracted watermark may vary from the original watermark [3].

\section{TYPES OF DIGITAL IMAGE WATERMARKING}

There are two types of watermarking techniques as follows:

- Visible watermarking

- Invisible watermarking

\subsection{Visible watermarking}

In this technique the embedded watermark is visible to the end user. It is the earlier technique of watermarking. The watermark is embedded on the cover page of the image. It is the first method of watermarking attempted for the purpose of security. It is embedded in such a way that it is clearly visible by the naked eyes to the user. In this watermark is added on the cover image. In this way watermark is visible to the end user clearly.

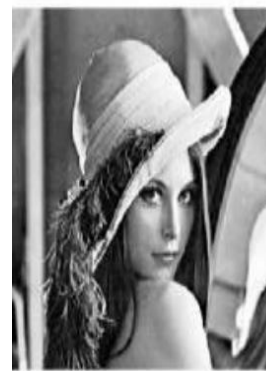

Original

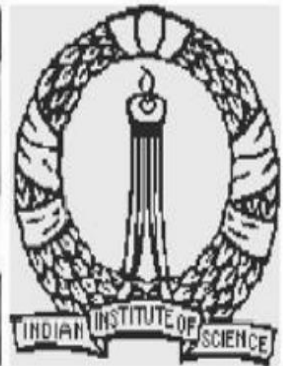

Watermark

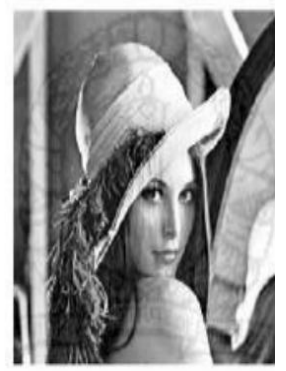

Watermarked Image
Figure1. Visible Watermark

\subsection{Invisible Watermarking}

Invisible Watermarking is that kind of watermarking technique in which embedded watermark is not visible to the viewer. In this the added watermark on the digital data cannot be perceive. The watermark is embedded on the overlaid image. This watermark is not visible to the end user but still it can be detected by using algorithms or various techniques. In this watermark is digitally embedded in the image. These kinds of watermarks are used to proof the ownership. It is also used to detect the misuse of the product. It is also considered as the backup for visible watermarking [2]. 


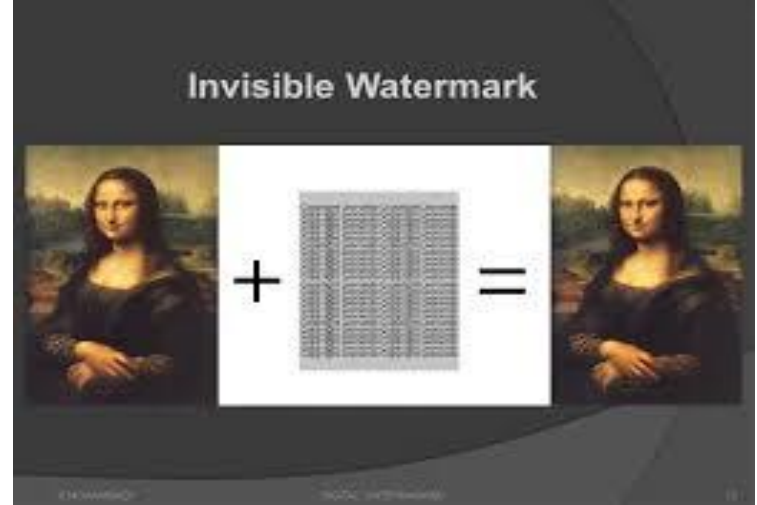

Fig 2 invisible watermark

\section{WATERMARKING TECHNIQUE}

In this section various trending watermarking techniques have been discussed.

1. DWT Transform: In this technique of watermarking image is subdivided into four parts. These are as horizontal part, diagonal part, vertical part, and approximation part. The image is divided into four parts for converting the image into low resolution image. The process is repeated for calculating the multiple scale wavelet decomposition. DWT is more preferable technique for watermarking because it performs computations very accurately. The positive point of this technique is that it is robust to handle the noise in the image.

2. DCT Transform: DCT stands for Discrete Cosine Transform. The main feature of using this technique is that it provides the good signal approximation by using certain coefficient values. This technique is used by many algorithms for embedding the watermarking on image. The main advantage of this technique is that it is quite fast as compare to other techniques. In this technique the watermark is embedded on the center frequency bands because of decomposition of the image. This technique is more robust to lossy compressions as compare to others.

3. DFT Transform: DFT i.e. Discrete Fourier Transform based watermarking technique, the brightness of the frame that is watermarked is obtained and the magnitude of the coefficients is taken to compute DFT. In this technique inverse DFT is also applied. This technique is robust and resistant to various attacks like pixel removal and rotation

\section{RELATED WORK}

i. Antonio Cedillo [1] et al., In this author explains that earlier the video watermarking was difficult to perform because of LSB algorithm. It is necessary to add watermark in the data to prevent it from unauthorized access. In this various techniques o9f watermarking is used based on several critwria like DCT, HVS, QIM, PSNR, SSIM.

ii. Mahima Jacob [2], et al In this author describes that digital watermarking becomes popular among users because it secures the digital data like audio, video from unauthorized access during the transmission over the network. Watermarking is a technique to protect digital data like audio, videos and textual data like documents from irrelevant access.

iii. Lalit Kumar Saini[3] et al in this author defines that it is the Era of internet over which the data is transferred. The data may be text, audio, video, image etc. In order to protect the data from tampering while transmission the technique of watermarking is developed. Watermarking use various algorithms to embed a watermark on the data.

iv. Rakesh Ahuja [4] et al., In this author defines the techniques of video watermarking. As it is the important issue concerned with security of digital data while transmission over the network. Watermarking is defined by two principles. Classification of digital data watermarking is based on various schemes. Various algorithms are used for digital data watermarking.

v. Monika Patel [5] et al. In this author describes that the digital data is easy to tamper and alter when tit is transmitted over the internet. To resolve this problem the concept of watermarking is developed. In watermarking the information related to the copyright or authentication is embedded on the original data which prevents the data from unauthorized access. Various algorithms can be used for embedding the watermark on the data. The selection of algorithm depends upon the nature of the data.

Bhattacharjee, T [6] et al..in this author defines atechnique which performs data hiding and sharing jointly. In this first of all image is divided into small shares and then these shares are embedded into the cover image for the purpose of hiding the data. Embedding of the data is done in DCT domain. It uses $\mathrm{m}$-array spread spectrum modulation. This is an analogous technique as $(\mathrm{k}, \mathrm{n})$ scheme in secret

vi. Sharing. On the bases of the number of users which are involved in decoding process, the quality access control can be accessed or obtained after simulation it is shown that the proposed technique is robust in nature and effective to the input or output of the system.

vii. Vinita Gupta [11] et al., in this paper it is defined that Digital watermarking is a process to hide the data behind any image, audio, video etc. It is a form of cryptography. In this paper image watermarking is explained. Various techniques are also discussed for image watermarking. Applications of image watermarking are also defined. Some factors and properties are also discussed along with a survey performed in New York on the basis of image watermarking.

viii. Preeti Parashar [13] et al, now a days mostly data travels over the internet for the purpose of communication. Thus the security of digital data is the main concern. In this author defines, that digital watermarking is a technique which is used to secure the confidential data. It prevents the data from duplication or tampering by hiding the secret message in the original information or data. The domains categorized the technique of image watermarking as spatial domain; transform domain etc. spatial domain is a technique which works on 
the basis of pixels. And the domain, frequency domain works on the transform coefficients o the image. In this paper author focuses on the spatial and transform domain along with their advantages and disadvantages.

\section{CONCLUSION}

Many papers are reviewed or seen regarding the watermarking and its techniques. All the techniques that are studied some of them are advancement in traditional techniques and some are bases for new proposed techniques. All the techniques are efficient and have many advantages but on other side there are some disadvantages also. There are many types of watermarking is available. Visible watermarking is clearly visible to the end user whereas invisible watermarking is not visible to the end users. Invisible watermarking can only be revealed by using some algorithms or techniques.

Further implementation can be done on this concept to provide security to the watermark image which is to embed in any media file. Security of the network can be enhancing with the combination of two or more techniques to prevent unauthorized access.

\section{REFERENCES}

[1] Jian Lu, 2014“A Robust Fractal Color Image Watermarking Algorithm"

[2] Mahima Jacob, Saurabh Mitra," Video Watermarking Techniques", IJRTE, Vol 4, Pp 1-4,2015.

[3] Lalit Kumar Saini, Vishal Shrivastava," A Survey of Digital Watermarking Techniques and its Applications", IJCST, Vol 2, Pp 70-73,2014

[4] Rakesh Ahuja, S. S. Bedi," All Aspects of Digital Video Watermarking Under an Umbrella", Ijigsp, Vol 12, Pp 54-73, 2015

[5] Monika Patel,Priti Srinivas Sajja," Analysis and Survey of Digital Watermarking Techniques", ijarcsse, Vol 3, Pp 203-210, 2013

[6] Bhattacharjee, T., Nov. 2014 "Progressive quality access through secret sharing and data hiding scheme"Pp 57,2014

[7] Radouane, M. April 2014 "Robust method of digital image watermarking using SVD transform on DWT coefficients with optimal block"

[8] Xie Yong,Oct. 2014 "Effect of Embedding Way on Printed Watermarking Image by Lithography"
[9] Saiyyad, M.A.M.,Dec. 2014 "Watermarking of compressed images using SWT technique and fragile approach "

[10] Yu Changhui, Oct. 2014, "Digital Watermarking Technology Based on DCT and Neural Net".

[11] Vinita Gupta , January 2014 "A Review on Image Watermarking and Its Techniques", I JMEIT ,Vol. 2, Issue 1, 2014

[12] M. Kim ,2014,“A Robust Digital Watermarking Technique for Image Contents based on DWT-DFRNT Multiple Transform Method"

[13] Preeti Parashar ,2014“A Survey: Digital Image Watermarking Techniques".

[14] Hussain Nyeem ,2014"Digital image watermarking: its formal model, fundamental properties and possible attacks"

[15] Bernd Girod, "Digital Watermarking of Text, Image, and Video Documents".

[16] Manjit Thapa, Sandeep Kumar Sood , "Digital watermarking is used to hide the information inside a signal.

[17] Sanjana Sinha , "Digital Video Watermarking using Discrete Wavelet Transform and Principal Component Analysis"

[18] G. G. Chiddarwar, The protection and illegal redistribution of digital media has become an important issue in the digital era.

[19] Loganathan Agilandeeswari1, 2013 "A Novel Block based Video in Video Watermarking Algorithm using Discrete Wavelet Transform and Singular Value Decomposition".

[20] M. Madhavi Latha,2010 ,"Watermarking of Digital Video Stream for Source Authentication", Vol. 7, Issue 4, No 1, July 2010

[21] Manjit Thapa , "Digital Image Watermarking Technique Based on Different Attacks".

[22] Mrs Neeta Deshpande ,2010“Review of Robust Video Watermarking Algorithms".

[23] Fadoua DRIRA, Image watermarking technique based on the steerable pyramid transform.

[24] Miroslav Goljan, "Comparing robustness of watermarking. 